\title{
OPEN Serum pentraxin 3 as a biomarker of hepatocellular carcinoma in chronic hepatitis B virus infection
}

\author{
Huan Deng ${ }^{1}$, Xiude Fan ${ }^{1}$, Xiaoyun Wang ${ }^{1}$, Lu Zeng ${ }^{1}$, Kun Zhang ${ }^{1}$, Xiaoge Zhang ${ }^{1}$, Na Li ${ }^{1}$, \\ Qunying $\operatorname{Han}^{1}, \mathrm{Yi} \mathrm{Lv}^{2,3}$ \& Zhengwen Liu' ${ }^{1,3 凶}$
}

Biomarkers for early diagnosis of hepatocellular carcinoma (HCC) are needed in chronic hepatitis B virus (HBV) infection, a leading cause of HCC. We evaluated whether measurement of serum pentraxin 3 (PTX3) could improve diagnosis of HCC in chronic HBV infection. Data from patients with HBVrelated chronic hepatitis $(n=159)$, cirrhosis $(n=99)$ and HCC $(n=107)$, and healthy controls $(n=151)$ were analyzed. Serum PTX3 concentration was measured by immunoassay. Area under the receiver operating characteristic curve (AUC) was applied to assess diagnostic accuracy. PTX 3 levels were significantly higher in HBV patients than in healthy controls $(P<0.001)$ and in HCC than in chronic hepatitis $(P<0.001)$ or cirrhosis patients $(P<0.001)$. PTX3 was an independent risk factor of HCC [odds ratio (OR) 1.617, $P<0.001$ ] and could distinguish HCC in chronic HBV infection [cutoff $9.231 \mathrm{ng} / \mathrm{mL}$, AUC 0.929 with $95 \%$ confidence interval (CI) of $0.898-0.953$ ], including $\alpha$-fetoprotein (AFP) negative [cutoff $8.985 \mathrm{ng} / \mathrm{mL}$, AUC $(95 \% \mathrm{Cl}) 0.947(0.908-0.973)$ ] and early-stage HCC [cutoff $9.359 \mathrm{ng} / \mathrm{mL}$, AUC $(95 \% \mathrm{Cl}) 0.920(0.885-0.947)]$. Combination of PTX3 with AFP improved the discrimination of early $\mathrm{HCC}$ from chronic HBV infection [AUC $(95 \% \mathrm{Cl}) 0.948(0.918-0.970)$ ]. In short, PTX3 measurement could identify HCC, including AFP-negative and early-stage HCC, in chronic HBV infection.

Hepatocellular carcinoma (HCC) is the sixth most common cancer and the second leading cause of cancer-related deaths worldwide with more than 840,000 new cases each year ${ }^{1}$. HCC is also the major cause of death among patients with cirrhosis ${ }^{2}$. Chronic hepatitis B virus (HBV) infection, which is associated with various liver diseases including chronic hepatitis, cirrhosis and $\mathrm{HCC}^{3-5}$, is the principal risk factor for HCC in East Asian counties including China ${ }^{6,7}$. The identification of HCC, especially at the early stage, from the various disease conditions in chronic HBV infection is of vital importance for improving patient prognosis because that early diagnosis is considered to be the only chance for long-term survival of HCC patients $^{7-9}$.

The commonly used modalities for detection of HCC include determination of serum alpha-fetoprotein (AFP), abdominal ultrasound, computed tomography and magnetic resonance imaging ${ }^{10}$. However, the differentiation of small HCC from cirrhotic nodules is still difficult by imaging. Meanwhile, AFP, the most commonly used biomarker in HCC, is not specific for HCC and elevated levels of AFP may be seen in both patients with HCC and chronic hepatitis without cancer ${ }^{11-13}$. In addition, AFP is potentially most suitable to detect advanced tumors because the concentrations are related to tumour size ${ }^{11}$. Moreover, many HCC patients may exhibit no elevation of AFP levels and AFP determination can not be utilized to detect HCC in these cases ${ }^{12}$. Therefore, identification of new biomarkers for diagnosis, especially early diagnosis, of HCC is an urgent need for clinical practice $^{14}$.

Pentraxin 3 (PTX3), also called tumor necrosis factor-stimulated gene 14 (TSG-14), is a long pentraxin of the pentraxin superfamily ${ }^{15,16}$, a class of ancient group of evolutionarily conserved and versatile proteins ${ }^{17,18}$. Structurally, PTX3 has an unrelated N-terminal domain linked to a pentraxin-like C-terminal domain ${ }^{19,20}$. PTX3 performs indispensable non-redundant roles in humoral innate immunity in microbial infections and acts as a connection between innate immunity, inflammation, tissue repair and cancer ${ }^{17,21-24}$. Notably, PTX3 is possibly involved in cancer development. For example, PTX3 expression levels have been shown to be related to the prognosis in certain types of cancer such as breast cancer ${ }^{25}$, gastric cancer ${ }^{26}$, lung carcinoma ${ }^{27}$, pancreatic carcinoma $^{28}$, and prostate cancer ${ }^{29}$.

\footnotetext{
${ }^{1}$ Department of Infectious Diseases, First Affiliated Hospital of Xi'an Jiaotong University, No. 277Yanta West Road, Xi'an 710061, Shaanxi Province, People's Republic of China. ${ }^{2}$ Department of Hepatobiliary Surgery, First Affiliated Hospital of Xi'an Jiaotong University, Xi'an 710061, Shaanxi, People's Republic of China. ${ }^{3}$ Institute of Advanced Surgical Technology and Engineering, Xi'an Jiaotong University, Xi'an 710061, Shaanxi, People's Republic of China. ${ }^{\square}$ email: liuzhengwen113@xjtu.edu.cn
} 
With regard to liver diseases, plasma PTX3 was indicated to be associated with nonalcoholic steatohepatitis $(\mathrm{NASH})^{30}$ and could differentiate NASH from non-NASH ${ }^{31}$. Elevated levels of PTX3 were also documented in acute liver injury caused by paracetamol and linked with adverse consequences in paracetamol overdose ${ }^{32}$. Hepatic and plasma PTX3 was raised in alcoholic hepatitis patients and PTX3 expression was related to the disease severity and short-term mortality ${ }^{33}$. Serum PTX3 was clinically shown to have valid diagnostic accuracy as a marker of fibrosis in chronic hepatitis $\mathrm{C}$ virus (HCV) infection ${ }^{34}$ and increased PTX3 plasma level was a risk factor for HCC occurrence in chronic HCV infection ${ }^{35}$. Furthermore, PTX3 was shown to be able to promote HCC progression and high PTX3 expression in tumor tissues was related to unfavorable prognosis in HCC patients ${ }^{36}$. Despite these studies, the potential role of circulating PTX3 levels in chronic HBV infection, especially HBV-related HCC, remains to be further examined. This study, therefore, was designed to determine the serum PTX3 levels in patients with various cross-sectional diseases including chronic hepatitis, cirrhosis and HCC during chronic HBV infection and to assess the potential diagnostic value in distinguishing HCC, including AFP-negative and early HCC, from other disease conditions in chronic HBV infection.

\section{Methods}

Study subjects. A total of 516 participants were included in this study. Among them, 365 were patients with chronic HBV infection (159 chronic hepatitis, 99 cirrhosis and 107 HBV-related HCC) and 151 were age- and sex-matched healthy controls. Chronic HBV infection and liver diseases were diagnosed in accordance with Clinical Practice Guidelines on the management of HBV infection ${ }^{37}$. Measurement of AFP level and ultrasound sonography were routinely performed in all the patients with chronic HBV infection. Chronic HBV infection was defined based on the presence of HBsAg for more than 6 months and detectable serum HBV DNA levels. Chronic hepatitis was diagnosed in view of abnormal biochemistry of liver function such as increased serum alanine aminotransferase (ALT) and aspartate aminotransferase (AST) levels in chronic HBV infection. Cirrhosis was determined based on imaging evidence of liver cirrhosis and existence of portal hypertension, ascites, varices and splenomegaly and/or changes of histopathology of liver biopsy samples with abnormal biochemistry of liver function such as increased ALT and AST levels and/or hypoalbuminia. HCC was determined on the base of characteristics of ultrasound, computed tomography and/or magnetic resonance and/or histopathology ${ }^{38}$. According to the commonly used upper limit of normal AFP level $(20 \mathrm{ng} / \mathrm{mL})^{38}$, the HCC was divided into AFP negative $(\leq 20 \mathrm{ng} / \mathrm{mL})$ or positive (AFP $>20 \mathrm{ng} / \mathrm{mL}$ ) HCC. HCC stage was defined in line with the Barcelona Clinic Liver Cancer (BCLC) staging system. The diseases were classified as early-stage (stage 0 and stage A) ${ }^{39}$ and late-stage (stage B, stage C and stage D) HCC. Patients with other liver disease such as nonalcoholic fatty liver disease, primary biliary cirrhosis, alcoholic liver disease and drug-induced liver injury were excluded. Patients with autoimmune diseases such as autoimmune hepatitis and systemic lupus erythematosus and severe diseases of other systems were also excluded. Patients aged under 18 years were also excluded. Blood samples were obtained from each participant. Serum was separated, aliquoted and stored at $-80^{\circ} \mathrm{C}$ until use.

Determination of laboratory parameters. Blood tests and liver functions were assayed at the central laboratory of the hospital. Serum HBV DNA was quantified by HBV quantitative polymerase chain reaction. $\mathrm{HBsAg}$, anti-HBs, $\mathrm{HBeAg}$, anti-HBe, and anti-HBc were determined by enzyme-linked immunosorbent assay. Serum AFP (ng/mL) was quantified by automated Eleceyes (Hoffman-La Roche Ltd., Basel, Switzerland).

Measurement of serum PTX3 concentration. Serum PTX3 concentration was estimated by The Quantikine Human Pentraxin 3/TSG-14 Immunoassay (R\&D Systems China Co., Ltd. Shanghai, China). The intra-assay precision [coefficient of variation $\mathrm{CV}(\%)$ ] is $3.6 \%$ to $4.4 \%$. The inter-assay precision is $4.1 \%$ to $6.2 \%$. The minimum detectable dose (MDD) of PTX3 is $0.007-0.116 \mathrm{ng} / \mathrm{mL}$. The mean MDD is $0.025 \mathrm{ng} / \mathrm{mL}$. No significant crossreactivity or interference was observed with human C-reactive protein and Pentraxin 2/Serum amyloid P component.

Statistical analysis. Statistical analysis was conducted using SPSS 24.0 software and MedCalc12.0 software. Quantitative variables were presented as mean and standard deviation (SD) or median and interquartile range. Categorical variables were expressed as absolute or relative frequencies. Continuous variables were compared using the analysis of variance. Categorical variables were compared by Chi-Square test. Risk factors for HCC were estimated using multivariable logistic regression analysis. The predictive values of relevant parameters were analyzed by receiver operating characteristic (ROC) curve to calculate the area under the curve (AUC), 95\% confidence interval (CI) and optimal cut-off value and its sensitivity, specificity, Yoden index, positive (PPV) and negative (NPV) predictive value, and positive and negative likelihood ratio (LR). Analysis of statistical differences between AUCs was performed using Z-test. To minimize the influence of data bias and confounding variables we also performed analysis of the data for patients with similar propensity scores using a 1:1 ratio matching after propensity score matching (PSM). Significance was defined as a two-tailed $P$ value less than 0.05 .

Ethical approval. This study was conducted in conform with the Declaration of Helsinki. Study approval was obtained from the Ethics Committee of the First Affiliated Hospital of Xi'an Jiaotong University. Each study participant gave informed consent. 


\begin{tabular}{|l|l|l|l|r|}
\hline & Chronic hepatitis $(\mathbf{n}=\mathbf{1 5 9})$ & Cirrhosis $(\mathbf{n}=\mathbf{9 9})$ & HCC $(\mathbf{n}=107)$ & \multicolumn{1}{l|}{} \\
\hline Gender $(\mathrm{male} / \mathrm{female})$ & $96 / 63$ & $61 / 38$ & $92 / 15$ & $<0.001$ \\
\hline Age $($ years $)$ & $32.30 \pm 12.39$ & $46.98 \pm 9.18$ & $49.44 \pm 10.90$ & $<0.001$ \\
\hline HBV DNA (IU/mL, log) & $5.92 \pm 1.83$ & $5.34 \pm 1.62$ & $4.90 \pm 1.50$ & $<0.001$ \\
\hline ALT $(\mathrm{IU} / \mathrm{L})$ & $81(31-331)$ & $48(28-84)$ & $54(33-102)$ & 0.003 \\
\hline AST $(\mathrm{IU} / \mathrm{L})$ & $65(29-152)$ & $58(37-83)$ & $70(35-150)$ & 0.538 \\
\hline TBIL $(\mu \mathrm{mol} / \mathrm{L})$ & $18.5(11.4-84.8)$ & $26.1(16.2-44.7)$ & $26.2(15.2-46.7)$ & 0.108 \\
\hline Albumin $(\mathrm{g} / \mathrm{L})$ & $38(34.9-41)$ & $30(26.8-36.2)$ & $33(29.7-39.1)$ & $<0.001$ \\
\hline AFP $(\mathrm{ng} / \mathrm{mL})$ & $12(6.8-26.2)$ & $12(4.82-42)$ & $97.4(16.8-4886)$ & $<0.001$ \\
\hline PTX3 $(\mathrm{ng} / \mathrm{mL})$ & $3.7(1.6-6.4)$ & $6.2(3-9)$ & $15.6(9.6-24.3)$ & $<0.001$ \\
\hline
\end{tabular}

Table 1. Demographic and laboratory parameters in the patients with different clinical diseases of chronic $\mathrm{HBV}$ infection. $H B V$ hepatitis B virus, HCC hepatocellular carcinoma, ALT alanine aminotransferase, AST aspartate aminotransferase, TBIL total bilirubin, PTX3 pentraxin 3, AFP a-fetoprotein.

\begin{tabular}{|c|c|c|c|c|c|c|c|c|c|}
\hline \multirow[b]{2}{*}{ Variable } & \multicolumn{3}{|c|}{$\begin{array}{l}\text { HCC versus } C H \\
(n=107) \text { versus }(n=159)\end{array}$} & \multicolumn{3}{|c|}{$\begin{array}{l}\text { HCC versus LC } \\
(n=107) \text { versus }(n=99)\end{array}$} & \multicolumn{3}{|c|}{$\begin{array}{l}\text { HCC versus } C H+L C \\
(n=107) \text { versus }(n=258)\end{array}$} \\
\hline & OR & SE & $p$ & OR & SE & $p$ & OR & SE & $\mathbf{P}$ \\
\hline Gender (Male/female) & 19.288 & 0.995 & 0.003 & 5.612 & 0.844 & 0.041 & 14.364 & 0.906 & 0.003 \\
\hline Age (years) & 1.137 & 0.032 & $<0.0001$ & 1.061 & 0.030 & 0.047 & 1.100 & 0.024 & $<0.0001$ \\
\hline HBV DNA (IU/mL, log) & 0.656 & 0.234 & 0.072 & 0.619 & 0.210 & 0.022 & 0.672 & 0.163 & 0.015 \\
\hline ALT (IU/L) & 0.996 & 0.004 & 0.243 & 0.999 & 0.005 & 0.767 & 0.997 & 0.003 & 0.303 \\
\hline AST (IU/L) & 1.003 & 0.004 & 0.421 & 1.002 & 0.003 & 0.570 & 1.002 & 0.002 & 0.388 \\
\hline TBIL $(\mu \mathrm{mol} / \mathrm{L})$ & 0.997 & 0.003 & 0.330 & 1.002 & 0.005 & 0.687 & 1.000 & 0.003 & 0.979 \\
\hline Albumin $(\mathrm{g} / \mathrm{L})$ & 0.995 & 0.061 & 0.930 & 0.909 & 0.043 & 0.026 & 0.942 & 0.038 & 0.120 \\
\hline PTX3 (ng/mL) & 1.639 & 0.097 & $<0.0001$ & 1.671 & 0.104 & $<0.0001$ & 1.617 & 0.075 & $<0.0001$ \\
\hline AFP $(\mathrm{ng} / \mathrm{mL})$ & 1.001 & 0.0002 & 0.014 & 1.003 & 0.002 & 0.193 & 1.001 & 0.0002 & 0.002 \\
\hline
\end{tabular}

Table 2. Factors associated with HCC by multivariate logistic regression. $H B V$ hepatitis B virus, $H C C$ hepatocellular carcinoma, $A L T$ alanine aminotransferase, $A S T$ aspartate aminotransferase, TBIL total bilirubin, $P T X 3$ pentraxin 3, AFP a-fetoprotein, $C H$ chronic hepatitis, $L C$ liver cirrhosis.

\section{Results}

Characteristics of the participants. This study recruited 516 participants including 365 patients with chronic HBV infection [male/female, 249/116; age, $41.31 \pm 13.69$ (18-78) years] and 151 healthy controls [male/ femal, 97/54; age, $42.28 \pm 14.13$ (18-76) years]. The male/female ratio and age between patients with chronic HBV infection and healthy controls were comparable $(P>0.05)$. Of the 365 patients, $159(43.6 \%)$ were diagnosed with chronic hepatitis, 99 (27.1\%) with cirrhosis and 107 (29.3\%) with HCC. The demographic and laboratory findings in the patients with different diseases were shown in Table 1.

Serum PTX3 levels in the study participants. Serum PTX3 levels in patients with chronic HBV infection $[6.63(3.02-10.24) \mathrm{ng} / \mathrm{mL}]$ were significantly increased compared with healthy controls $[1.03(0.6-1.7) \mathrm{ng} /$ $\mathrm{mL}, P<0.001]$ (Figure S1A). HCC patients had significantly increased PTX3 levels [15.6 $(9.6-24.3) \mathrm{ng} / \mathrm{mL}]$ compared with patients with chronic hepatitis [3.7 (1.6-6.4) ng/mL, $P<0.001$, Figure S1B], cirrhosis [6.2 (3-9) $\mathrm{ng} / \mathrm{mL}, P<0.001$, Figure S1B,] or chronic HBV infection without HCC [chronic hepatitis + cirrhosis, 4.2 (2-7.9) $\mathrm{ng} / \mathrm{mL}, P<0.001$, Figure $\mathrm{S} 1 \mathrm{C}]$.

According to underlying liver disease, patients with chronic hepatitis and HCC $(n=23)$ had significantly higher PTX3 levels than patients with chronic hepatitis without HCC $(\mathrm{n}=159)$ [13.3 (6.8-38.8) ng/mL vs. $3.7(0.089-22.37) \mathrm{ng} / \mathrm{mL}, P<0.001$, Figure S2A]. Patients with cirrhosis and HCC $(\mathrm{n}=84)$ also had significantly higher PTX3 levels than patients with cirrhosis without HCC $(\mathrm{n}=99)[15.9(1.3-56.6) \mathrm{ng} / \mathrm{mL}$ vs. 6.18 (0.83-14.02) ng/mL, $P<0.001$, Figure S2B].

Association of PTX3 levels with HCC in chronic HBV infection. The patients were categorized as chronic hepatitis, cirrhosis and chronic HBV infection without HCC (chronic hepatitis plus cirrhosis). Multivariate logistic regression on factors for HCC showed that, in comparison with chronic hepatitis, in addition to gender (OR 19.288, $P=0.003$ ), age (OR 1.137, $P<0.001$ ) and AFP (OR 1.001, $P=0.014$ ), PTX3 (OR 1.639, $P<0.001$ ) was an independent risk factor for HCC (Table 2). In relation to cirrhosis, together with gender (OR 5.612, $P=0.041$ ), age (OR 1.061, $P=0.047$ ), HBV DNA (OR 0.619, $P=0.022$ ) and albumin (OR 0.909, $P=0.026)$, PTX3 (OR 1.671, $P<0.001$ ) was an independent risk factor for HCC (Table 2). In relation to HBV infection without HCC, in addition to gender (OR 14.364, $P=0.003$ ), age (OR $1.100, P<0.001$ ), HBV DNA (OR 0.672, 
A

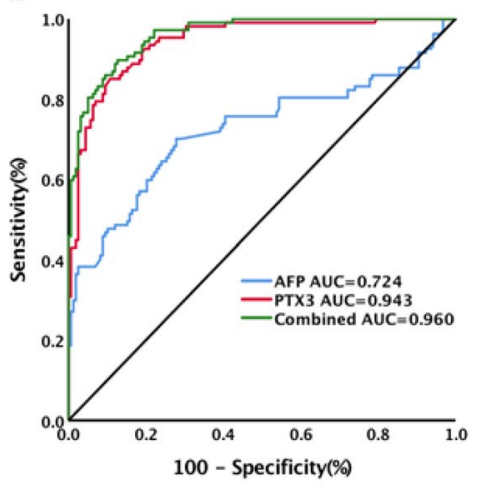

B

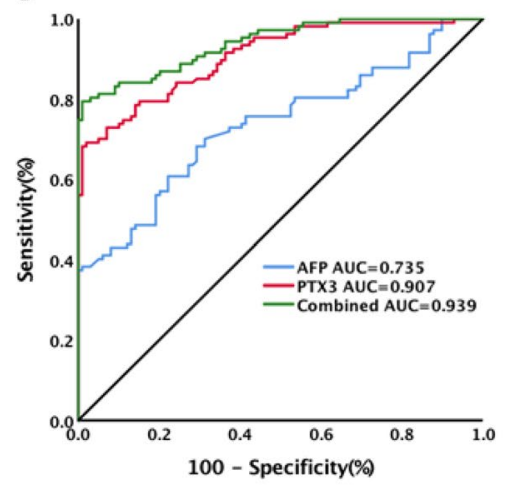

C

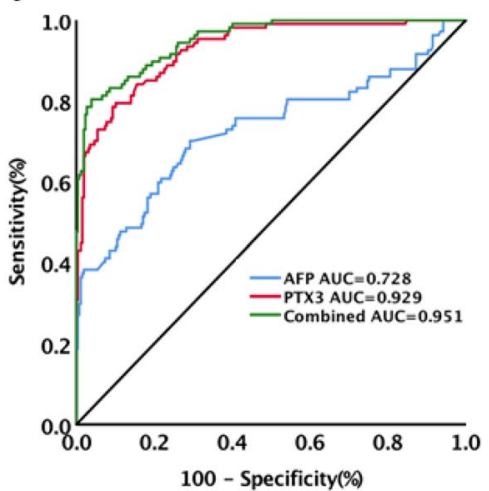

Figure 1. Diagnostic performance of serum PTX3, AFP and combination of PTX3 with AFP for hepatocellular carcinoma (HCC) in patients with chronic HBV infection. (A) Diagnostic performance of serum PTX3, AFP and combination of PTX3 with AFP for HCC from chronic hepatitis; (B) Diagnostic performance of serum PTX3, AFP and combination of PTX3 with AFP for HCC from cirrhosis; (C) Diagnostic performance of serum PTX3, AFP and combination of PTX3 with AFP for HCC from patients without HCC (chronic hepatitis and cirrhosis).

$P=0.015)$ and AFP (OR 1.001, $P=0.002$ ), PTX3 (OR 1.617, $P<0.001$ ) was also an independent risk factor for HCC (Table 2).

Of the HCC patients, 43 patients had histological results of HCC. Of the 43 patients, 17 had low differentiation, 19 had moderate differentiation and 7 had high differentiation. The PTX3 levels in patients with low differentiation were significantly higher than in patients with high differentiation [20.57(8.47-56.62) ng/ $\mathrm{mL}$ vs. 7.93(5.95-9.03) ng/mL, $P<0.001]$. Patients with moderate differentiation also had higher PTX3 levels than patients with high differentiation $[15.10(7.78-42.18) \mathrm{ng} / \mathrm{mL}$ vs. $7.93(5.95-9.03) \mathrm{ng} / \mathrm{mL}, P=0.001]$. The PTX3 levels in patients with low differentiation were higher than in patients with moderate differentiation [20.57(8.47-56.62) ng/mL vs. $15.10(7.78-42.18) \mathrm{ng} / \mathrm{mL}]$ although the difference was not significant $(P=0.383)$.

Diagnostic performance of serum PTX3 levels for HCC. The performance of PTX3 for identifying HCC was assessed. Serum PTX3 levels could significantly differentiate HCC from chronic hepatitis [AUC (95\% CI) 0.943 (0.908-0.968), sensitivity $85.1 \%$, specificity 89.3\%, Fig. 1A], cirrhosis [AUC (95\% CI) 0.907 (0.859-0.943), sensitivity $68.2 \%$, specificity $99.0 \%$, Fig. 2B] or chronic HBV infection without HCC [AUC (95\% CI) 0.929 (0.898-0.953), sensitivity 79.4\%, specificity 89.9\%, Fig. 2C, Table 3 ). The corresponding results of AFP were: HCC versus chronic hepatitis: AUC (95\% CI) 0.724 (0.666-0.776), sensitivity 70.1\%, specificity $72.3 \%$ (Fig. 2A); HCC versus cirrhosis: AUC (95\% CI) 0.735 (0.670-0.794), sensitivity $68.2 \%$, specificity $70.7 \%$ (Fig. 1B); and HCC versus chronic HBV infection without HCC: AUC (95\%CI) 0.728 (0.679-0.773), sensitivity $70.1 \%$, specificity $70.5 \%$ (Fig. 1C). The combination of PTX3 with AFP improved the performance of PTX3 or AFP alone. The corresponding results of combination of PTX3 with AFP were: HCC versus chronic hepatitis: AUC (95\%CI) 0.960 (0.929-0.980), sensitivity 89.7\%, specificity 87.4\% (Fig. 1A); HCC versus cirrhosis: AUC (95\%CI) 0.939 (0.898-0.968), sensitivity 79.4\%, specificity 99.0\% (Fig. 1B); and HCC versus chronic HBV infection without HCC: AUC (95\%CI) 0.951 (0.924-0.971), sensitivity $80.4 \%$, specificity $96.1 \%$ (Fig. 1C, Table 3 ).

After PSM, all the parameters (except PTX3 levels) in HCC $(\mathrm{n}=44)$ versus chronic hepatitis $(\mathrm{n}=44)$, HCC $(n=61)$ versus cirrhosis $(n=61)$ and HCC $(n=80)$ versus non-HCC $(n=80)$ were well matched (Table S1). Analysis of the data after PSM yielded similar results. The AUCs in HCC versus chronic hepatitis, HCC versus cirrhosis and HCC versus non-HCC were 0.954 (0.887-0.987), 0.914 (0.849-0.957) and 0.936 (0.887-0.969), respectively (Table S2).

Among the patients with chronic HBV infection, 87 patients received antiviral treatment with nucleos(t)ide analogues for more than six months and 278 did not receive antiviral treatment (Table S3). PTX3 levels could highly discriminate HCC in patients under or not under antiviral treatment. In patients under antiviral treatment, PTX3 levels had an AUC (95\%CI) of 0.923 (0.865-0.982), sensitivity of 80\%, and specificity of $89.47 \%$ for HCC in chronic HBV infection without HCC. AFP levels had an AUC (95\%CI) of 0.731 (0.609-0.852), sensitivity of $43.33 \%$, and specificity of $100 \%$. The combination of PTX3 and AFP had an AUC (95\%CI) of 0.947 (0.900-0.994), sensitivity of $76.67 \%$ and specificity of $98.25 \%$ (Figure S3A, Table S4). In patients not under antiviral treatment, PTX3 levels had an AUC (95\%CI) of 0.929 (0.896-0.963), sensitivity of 79.22\%, and specificity of 91.54\%. AFP levels had an AUC (95\%CI) of 0.729 (0.653-0.804), sensitivity of $68.83 \%$ and specificity of $74.13 \%$. The combination of PTX3 and AFP had an AUC (95\%CI) of 0.952 (0.927-0.977), sensitivity of $80.52 \%$ and specificity of 96.02\% (Figure S3B, Table S4).

According to AFP levels (Table S5), PTX3 levels remained highly discriminative of AFP negative HCC from chronic hepatitis [AUC (95\%CI) 0.956 (0.909-0.983), sensitivity 90.6\%, specificity 91.2\%, Fig. 2A], cirrhosis [AUC (95\%CI) $0.933(0.865-0.973)$, sensitivity $81.3 \%$, specificity $95.6 \%$, Fig. 2 B] and chronic HBV infection without HCC [AUC (95\%CI) 0.947 (0.908-0.973), sensitivity $90.6 \%$, specificity $86.1 \%$, Fig. 2C, Table S6]. The corresponding results of AFP were: HCC versus chronic hepatitis: AUC (95\%CI) 0.625 (0.540-0.704), sensitivity $40.6 \%$, specificity $86.7 \%$ (Fig. 2A); HCC versus cirrhosis: AUC (95\%CI) 0.531 (0.429-0.632), sensitivity 


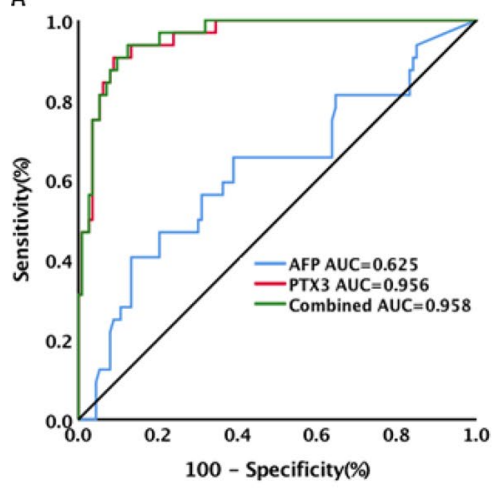

D

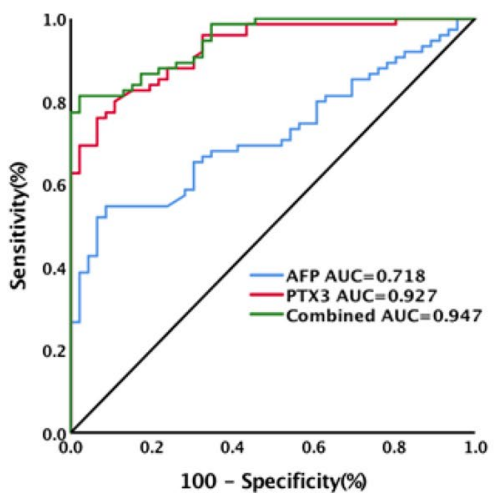

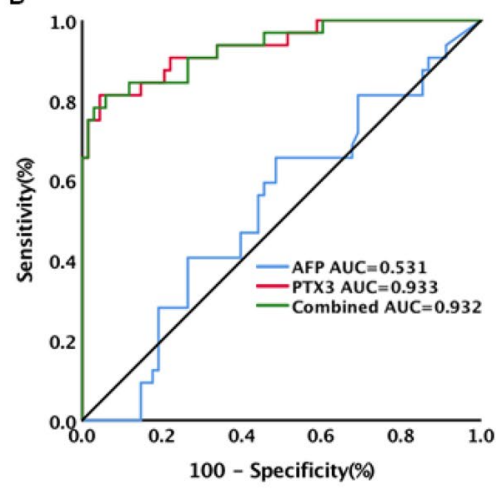

E

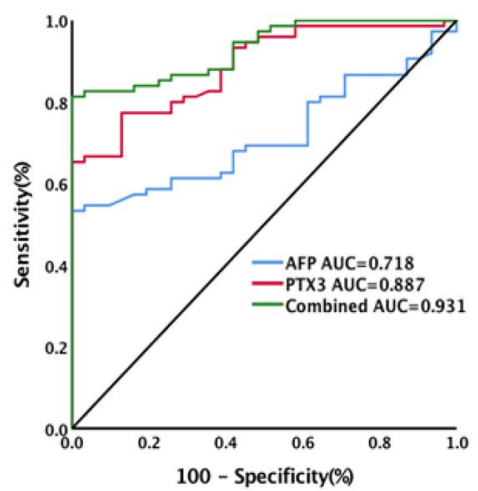

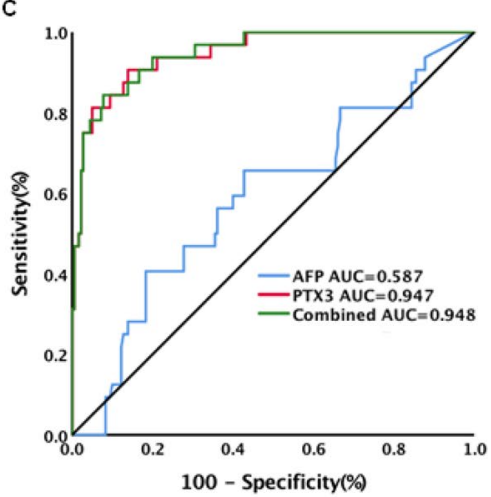

F

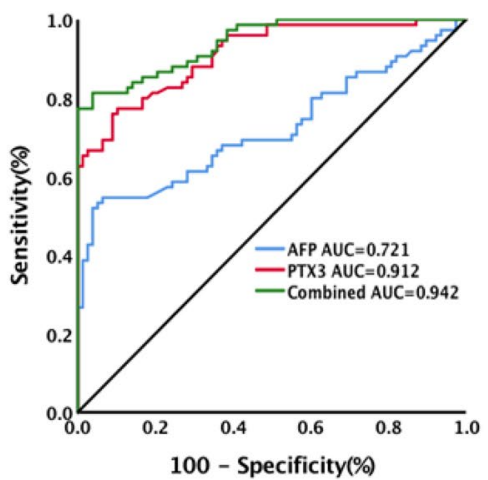

Figure 2. Diagnostic performance of serum PTX3, AFP and combination of PTX3 with AFP for AFP negative $(\leq 20 \mathrm{ng} / \mathrm{mL})$ and positive $(>20 \mathrm{ng} / \mathrm{mL}$ ) hepatocellular carcinoma (HCC) in patients with chronic HBV infection. (A) Diagnostic performance of serum PTX3, AFP and combination of PTX3 with AFP for AFP negative HCC from chronic hepatitis; (B) Diagnostic performance of serum PTX3, AFP and combination of PTX3 with AFP for AFP negative HCC from cirrhosis; (C) Diagnostic performance of serum PTX3, AFP and combination of PTX3 with AFP for AFP negative HCC from patients without HCC (chronic hepatitis and cirrhosis); (D) Diagnostic performance of serum PTX3, AFP and combination of PTX3 with AFP for AFP positive HCC from chronic hepatitis; (E) Diagnostic performance of serum PTX3, AFP and combination of PTX3 with AFP for AFP positive HCC from cirrhosis; (F) Diagnostic performance of serum PTX3, AFP and combination of PTX3 with AFP for AFP positive HCC from patients without HCC (chronic hepatitis and cirrhosis).

65.6\%, specificity 51.5\% (Fig. 2B); and HCC versus chronic HBV infection without HCC: AUC (95\%CI) 0.587 (0.518-0.654), sensitivity 65.6\%, specificity $57.2 \%$ (Fig. 2C, Table S4). The addition of AFP to PTX3 did not significantly improve the performance of PTX3 for discriminating AFP negative HCC. Analysis in patients with AFP positive (> $20 \mathrm{ng} / \mathrm{mL}$ ) HCC showed that PTX3 levels were also highly discriminative of HCC from chronic hepatitis [AUC (95\%CI) 0.927 (0.865-0.966), sensitivity 76\%, specificity 93.5\%, Fig. 2D], cirrhosis [AUC (95\%CI) 0.887 (0.810-0.940), sensitivity $65.3 \%$, specificity $100 \%$, Fig. 2E] and chronic HBV infection without HCC [AUC (95\%CI) 0.912 (0.855-0.952), sensitivity 77.3\%, specificity $89.7 \%$, Fig. 2E, Table S6]. The corresponding results of AFP were: HCC versus chronic hepatitis: AUC (95\%CI) 0.718 (0.629-0.796), sensitivity 54.7\%, specificity 91.3\% (Fig. 2D); HCC versus cirrhosis: AUC (95\%CI) 0.718 (0.622-0.810), sensitivity 53.3\%, specificity $100 \%$ (Fig. 2E); and HCC versus chronic HBV infection without HCC: AUC (95\%CI) 0.721 (0.643-0.790), sensitivity $54.7 \%$, specificity $93.6 \%$ (Fig. 2F, Table S6). The addition of AFP to PTX3 significantly increased the performance of PTX3 for discriminating AFP positive HCC from cirrhosis [AUC (95\%CI) 0.931 (0.865-0.971), sensitivity $81.33 \%$, specificity $100 \%$, Fig. 2E] or chronic HBV infection without HCC [AUC (95\%CI) 0.942 (0.892-0.973), sensitivity $81.3 \%$, specificity $96.2 \%$, Fig. 2F, Table S6].

According to HCC stages (Table S7), PTX3 levels also remained highly discriminative of early-stage HCC from chronic hepatitis [AUC (95\%CI) 0.935 (0.894-0.963), sensitivity 94.1\%, specificity 79.3\%, Fig. 3A], cirrhosis [AUC (95\%CI) 0.897 (0.840-0.938), sensitivity 69.1\%, specificity 98.0\%, Fig. 3B] and chronic HBV infection without HCC [AUC (95\%CI) 0.920 (0.885-0.947), sensitivity 76.5\%, specificity 90.70\%, Fig. 3C, Table S8]. The corresponding results of AFP were: HCC versus chronic hepatitis: AUC (95\%CI) 0.665 (0.599-0.726), sensitivity $64.7 \%$, specificity $72.3 \%$ (Fig. 3A); HCC versus cirrhosis: AUC (95\%CI) 0.679 (0.603-0.749), sensitivity $63.2 \%$, specificity $70.7 \%$ (Fig. 3B); and HCC versus chronic HBV infection without HCC [AUC (95\%CI) 0.670 (0.616-0.721), sensitivity 64.7\%, specificity 70.5\%, Fig. 3C, Table S8). The combination of PTX3 with AFP did not improve the performance of PTX3 for differentiating early-stage HCC from chronic hepatitis (AUC 0.957, Fig. 3A) but improved the performance for discriminating early-stage HCC from cirrhosis [AUC (95\%CI) 0.936 (0.888-0.968), sensitivity 79.4\%, specificity 98\%, Fig. 3C] or chronic HBV infection without HCC [AUC (95\%CI) 


\begin{tabular}{|c|c|c|c|c|c|c|c|c|c|}
\hline & $\begin{array}{l}\text { AUC }(95 \% \\
\text { CI) }\end{array}$ & $\begin{array}{l}\text { Cutoff (ng/ } \\
\mathrm{mL} \text { ) }\end{array}$ & $\begin{array}{l}\text { Sensitivity } \\
(\%)\end{array}$ & $\begin{array}{l}\text { Specificity } \\
(\%)\end{array}$ & $\begin{array}{l}\text { Youden } \\
\text { index }\end{array}$ & PPV (\%) & NPV (\%) & Positive LR & $\begin{array}{l}\text { Negative } \\
\text { LR }\end{array}$ \\
\hline \multicolumn{10}{|l|}{$\begin{array}{l}\mathrm{HCC} \text { versus } \\
\mathrm{CH}\end{array}$} \\
\hline PTX3 & \begin{tabular}{|l|}
0.943 \\
$(0.908-$ \\
$0.968)$
\end{tabular} & 8.949 & 85.1 & 89.3 & 0.744 & 84.3 & 89.9 & 7.95 & 0.17 \\
\hline AFP & \begin{tabular}{|l|}
0.724 \\
$(0.666-$ \\
$0.776)$
\end{tabular} & 20.36 & 70.1 & 72.3 & 0.424 & 63 & 78.2 & 2.53 & 0.41 \\
\hline PTX3 + AFP & \begin{tabular}{|l|}
0.960 \\
$(0.929-$ \\
$0.980)$
\end{tabular} & - & 89.7 & 87.4 & 0.771 & 82.8 & 92.7 & 7.13 & 0.12 \\
\hline \multicolumn{10}{|l|}{$\begin{array}{l}\text { HCC versus } \\
\text { LC }\end{array}$} \\
\hline PTX3 & \begin{tabular}{|l|}
0.907 \\
$(0.859-$ \\
$0.943)$ \\
\end{tabular} & 11.719 & 68.2 & 99.0 & 0.672 & 98.6 & 74.2 & 67.54 & 0.32 \\
\hline AFP & \begin{tabular}{|l|}
0.735 \\
$(0.670-$ \\
$0.794)$
\end{tabular} & 22.22 & 68.2 & 70.7 & 0.389 & 71.6 & 67.3 & 2.33 & 0.45 \\
\hline PTX3 + AFP & \begin{tabular}{|l|}
0.939 \\
$(0.898-$ \\
$0.968)$
\end{tabular} & - & 79.4 & 99.0 & 0.784 & 98.8 & 81.7 & 78.6 & 0.21 \\
\hline \multicolumn{10}{|l|}{$\begin{array}{l}\text { HCC versus } \\
\mathrm{CH}+\mathrm{LC}\end{array}$} \\
\hline PTX3 & \begin{tabular}{|l|}
0.929 \\
$(0.898-$ \\
$0.953)$
\end{tabular} & 9.231 & 79.4 & 89.9 & 0.693 & 76.6 & 91.3 & 7.88 & 0.23 \\
\hline AFP & \begin{tabular}{|l|}
0.728 \\
$(0.679-$ \\
$0.773)$
\end{tabular} & 20.36 & 70.1 & 70.5 & 0.406 & 49.7 & 85 & 2.38 & 0.42 \\
\hline PTX3 + AFP & \begin{tabular}{|l|}
0.951 \\
$(0.924-$ \\
$0.971)$
\end{tabular} & - & 80.4 & 96.1 & 0.765 & 89.6 & 92.2 & 20.74 & 0.2 \\
\hline
\end{tabular}

Table 3. Diagnostic performance of serum PTX3, AFP and the combination of PTX3 with AFP levels for HCC in chronic hepatitis, cirrhosis and chronic HBV infection without $\mathrm{HCC}(\mathrm{CH}+\mathrm{LC})$. PTX3 pentraxin 3 , $A F P$ alpha-fetoprotein, $H B V$ hepatitis B virus, $H C C$ hepatocellular carcinoma, $C H$ chronic hepatitis, $L C$ liver cirrhosis, $A U C$ area under receiver operating characteristic (ROC) curve, $P P V$ positive predictive value, $N P V$ negative predictive value, $L R$ likelihood ratio.

0.948 (0.918-0.970), sensitivity 79.4\%, specificity 96.1\%, Fig. 3C, Table S6]. For late-stage HCC, PTX3 levels also remained highly discriminative of HCC from chronic hepatitis [AUC (95\%CI) 0.958 (0.920-0.981), sensitivity 89.7\%, specificity 89.3\%, Fig. 3D], cirrhosis [AUC (95\%CI) 0.924 (0.867-0.962), sensitivity 84.6\%, specificity 84.9\%, Fig. 3E] and chronic HBV infection without HCC [AUC (95\%CI) 0.945 (0.913-0.968), sensitivity 84.6\%, specificity $89.9 \%$, Fig. 3F, Table S8]. The corresponding results of AFP were: HCC versus chronic hepatitis: AUC (95\%CI) 0.826 (0.766-0.876), sensitivity 79.5\%, specificity 72.3\% (Fig. 3D); HCC versus cirrhosis: AUC (95\%CI) 0.833 (0.760-0.891), sensitivity 56.4\%, specificity $95.0 \%$ (Fig. 3E); and HCC versus chronic HBV infection without HCC: AUC (95\%CI) 0.829 (0.781-0.870), sensitivity 69.2\%, specificity $81.8 \%$ (Fig. 3F, Table S8). The combination of PTX3 with AFP, although slightly increased AUC values, did not significantly increase the performance of PTX3 for discriminating late-stage HCC from chronic hepatitis (AUC 0.965), cirrhosis (AUC 0.944) or chronic HBV infection without HCC (AUC 0.956, Fig. 3, Table S8).

\section{Discussion}

This study demonstrated significantly elevated serum PTX3 levels in patients with chronic HBV infection compared to healthy controls and in patients with HBV-related HCC compared to patients with chronic hepatitis or cirrhosis. High PTX3 level was an independent risk factor of HCC. The PTX3 levels in HCC patients appeared to be related to HCC differentiation, with low differentiation HCC having higher PTX3 levels in comparison to high differentiation HCC. Importantly, serum PTX3 levels could highly discriminate HCC from chronic hepatitis, cirrhosis and chronic HBV infection without HCC. The discrimination of HCC by PTX3 levels was not influenced by the underlying disease (chronic hepatitis or cirrhosis) or the usage of antiviral treatment with nucleos(t)ide analogues. In particular, PTX3 levels were highly discriminative of AFP-negative $(\leq 20 \mathrm{ng} / \mathrm{mL})$ and early-stage HCC from chronic hepatitis, cirrhosis and chronic HBV infection without HCC. These findings suggest that PTX3 may act as a major contributing factor to the progress of HBV disease and the development of HCC. The determination of PTX3 levels may have a significant diagnostic value for HBV-related HCC including AFP-negative and early-stage HCC in chronic HBV infection.

PTX3 has been revealed to be an extrinsic oncosuppressor in preclinical models and certain tumors through regulating complement-driven macrophage-mediated tumor progression and tuning cancer-related 
A

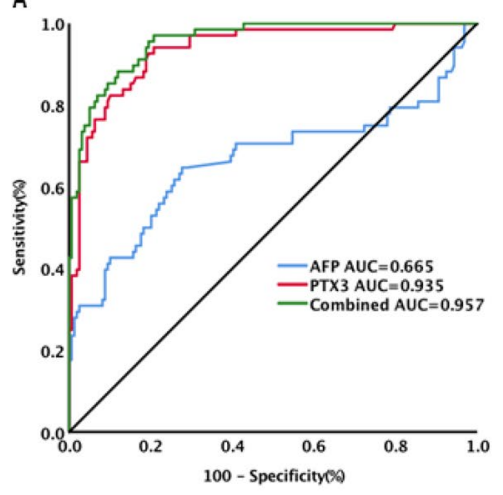

D

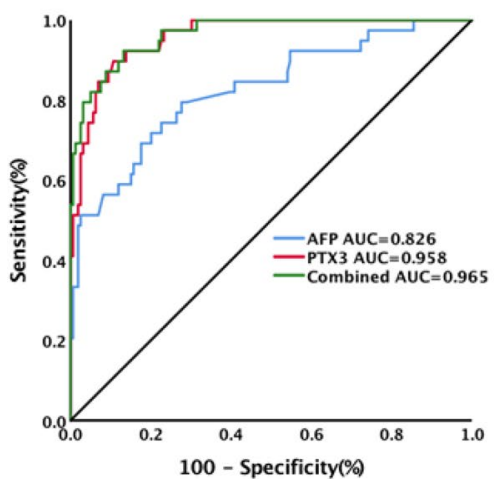

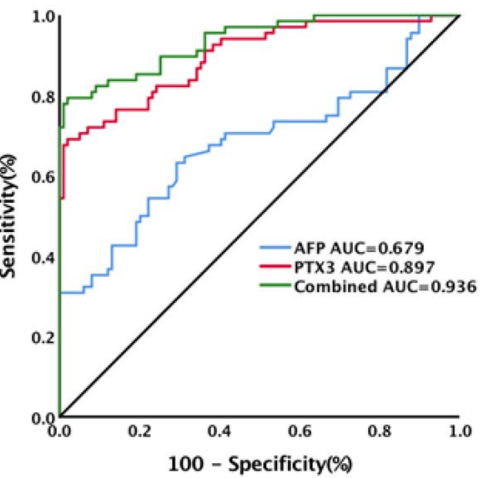

E

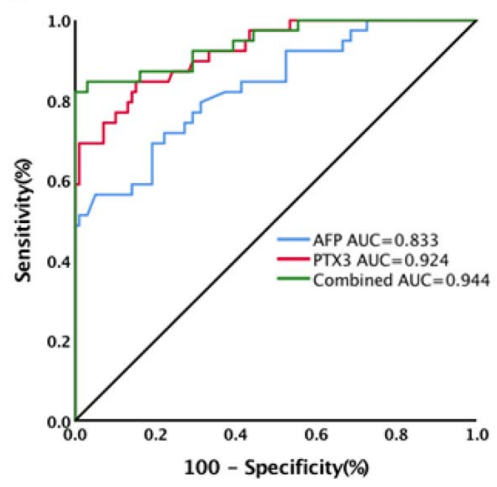

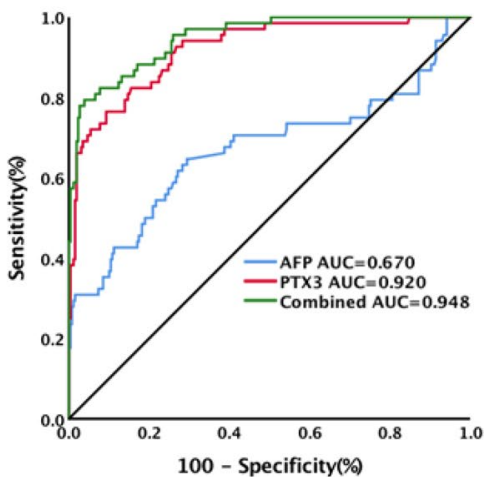

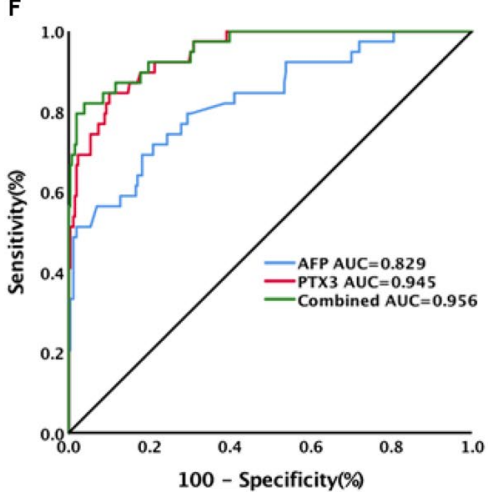

Figure 3. Diagnostic performance of serum PTX3, AFP and combination of PTX3 with AFP for early and late hepatocellular carcinoma (HCC) in patients with chronic HBV infection. (A) Diagnostic performance of serum PTX3, AFP and combination of PTX3 with AFP for early HCC from chronic hepatitis; (B) Diagnostic performance of serum PTX3, AFP and combination of PTX3 with AFP for early HCC from cirrhosis; (C) Diagnostic performance of serum PTX3, AFP and combination of PTX3 with AFP for early HCC from patients without HCC (chronic hepatitis and cirrhosis); (D) Diagnostic performance of serum PTX3, AFP and combination of PTX3 with AFP for late HCC from chronic hepatitis; (E) Diagnostic performance of serum PTX3, AFP and combination of PTX3 with AFP for late HCC from cirrhosis; (F) Diagnostic performance of serum PTX3, AFP and combination of PTX3 with AFP for late HCC from patients without HCC (chronic hepatitis and cirrhosis).

inflammation $22,25,40,41$. However, it remains unknown whether PTX3 plays a protective or promotive role in cancer in that it may impose an important influence on various aspects of cancer such as tumor initiation, angiogenesis, metastasis and immune-regulation ${ }^{42}$. Elevated PTX3 expression has been shown to be associated with poor prognosis in certain cancers, such as breast cancer ${ }^{25}$, gastric cancer ${ }^{26}$, lung cancer ${ }^{27}$, pancreatic cancer ${ }^{28}$ and prostate cancer ${ }^{29}$. Elevated PTX3 plasma level was a risk factor for HCC occurrence in chronic HCV infection ${ }^{35}$ and higher PTX3 expression in tumor tissues was also related to poor prognosis in HCC patients ${ }^{36}$. Consistently, this study showed that elevated circulating PTX3 levels were related to HCC development in chronic HBV infection.

The mechanisms by which PTX3 could be related to the occurrence and development of HCC are largely unknown. There are several potential explanations. First, PTX3 plays a complicated regulatory role in cancerrelated inflammation ${ }^{22,24}$. In liver diseases, PTX3 levels were markedly higher in NASH than in simple steatosis non-NASH patients ${ }^{30,31}$. PTX3 levels were also related to the severity of liver fibrosis in NASH ${ }^{30}$ and HCV infection ${ }^{34}$. It is suggested that PTX3 is involved in pathogenesis of hepatic inflammation and fibrosis which are common milieu at the origin of $\mathrm{HCC}^{43}$. Second, the involvement of PTX3 in immune response may also play a role in its association with HCC. Expression of PTX3 is inducible by tumor necrosis factor (TNF)- $\alpha$ and interleukin (IL)-1 ${ }^{44}$. Differentialy expressed TNF- $\alpha$ from HBV-specific CD8 + T cells was associated with the outcomes of chronic HBV infection including asymptomatic status, active chronic hepatitis and HBV-related $\mathrm{HCC}^{45}$. IL-1 $\beta$ levels were significantly and progressively increased with the disease advancement to HCC in chronic HBV infection ${ }^{46}$. Additionally, IL-10 could stimulate B cells in adaptive immunity through enhancing PTX3 production ${ }^{20}$. Elevated IL-10 was suggested to mediate disease progress, from inactive state to cirrhosis and HCC in HBV infection ${ }^{47}$. HCC patients also exhibited significantly higher frequencies of IL-10-expressing B cells that could suppress cytotoxic CD4+ T cell function related to poor survival and high recurrence rate of $\mathrm{HCC}^{48}$. Third, although PTX3 is believed not to be produced by hepatocytes ${ }^{49}$, it was shown to be able to enhance HCC cell proliferation and to induce epithelial-mesenchymal transition $(\mathrm{EMT})^{36}$, a biologic process closely related to tumor cell invasion and metastasis.

An important factor for improving long-term prognosis of HCC patients is to detect and treat the tumor at its early stage $\mathrm{e}^{7,9}$. AFP is the most commonly used biomarker but it is not suitable to detect early stage ${ }^{11}$ and 
AFP-negative $\mathrm{HCC}^{12}$. Therefore, identification of novel biomarkers for HCC remains an urgent need. In the present study, PTX3 was highly discriminative of HCC in chronic HBV infection. In particular, PTX3 was highly and accurately discriminative of AFP-negative and early-stage HCC and the diagnostic performance of PTX3 was superior to AFP. These findings suggest the potentail of PTX3 to be used as a more sensitive biomarker for HCC including early HCC and as a supplemental biomarker for AFP-negative or low AFP-displaying HCC in chronic HBV infection.

For diagnostic accuracy, PTX3 exhibited higher AUC, sensitivity, and specificity than did AFP in HCC patients in relation to chronic hepatitis, cirrhosis and chronic HBV infection without HCC. Detecting of both PTX3 and AFP improved the diagnostic accuracy for HCC in comparison with either detection alone. Although the addition of AFP to PTX3 did not significantly increase the diagnostic ability of PTX3 alone for AFP-negative HCC, the combination of PTX3 with AFP improved diagnostic accuracy when only early-stage HCC was evaluated.

Addition of AFP to ultrasound was shown to significantly increase sensitivity of early HCC detection ${ }^{50}$. PTX3 exhibited good diagnostic performance for the detection of early HCC from HBV chronically infected populations. Whether addition of PTX3 to ultrasound can improve the sensitivity of HCC detection deserves further investigation. Moreover, this study showed that the addition of PTX3 to AFP may allow further identification of HCC in the HBV-related liver diseases. Whether addition of both PTX3 and AFP to ultrasound can further improve the ability and accuracy of HCC detection also deserves investigation. Moreover, serum des-gammacarboxy prothrombin (DCP) was revealed to be an ideal marker for the diagnosis of HBV-related $\mathrm{HCC}^{51}$. We did not compare PTX3 and DCP in the study because measurement of DCP was not carried out in this study. The comparison of PTX3 and DCP is an interesting issue to be addressed in future studies.

We recognized some limitations of the study. First, this is a retrospective study in a relatively small number of patient population with cross-sectional diseases of chronic HBV infection and did not examine the changes of PTX3 levels before and after HCC development. Second, the lack of a validation population may affect the strength of the study. Third, this study only included patients with chronic HBV infection and patients with other etiologies were not included for investigation and comparison. This limits the generalization of the findings to disease conditions of other etiologies such as chronic HCV infection and NASH. Nevertheless, this study demonstrated consistent findings of the diagnostic accuracy of PTX3 for HCC in various disease conditions of chronic HBV infection, and both multivariable analysis and the analysis of data after PSM exhibited the independent role and diagnostic potential of PTX3 in HBV-related HCC, providing evidence for further studies of enhancing and extending the diagnostic potential of PTX3 in HCC detection and optimization of HCC surveillance.

\section{Conclusion}

This study demonstrated the differences of circulating PTX3 levels in various diseases in chronic HBV infection and identified serum PTX3 as an independent factor associated with HCC. Particularly, PTX3 can be used as a new indicator for HCC diagnosis. The performance of PTX3 for differentiating HCC from chronic HBV infection is superior to AFP. Furthermore, PTX3 is highly and accurately discriminative of AFP-negative and earlystage HCC. The combination of PTX3 with AFP may allow further identification of HCC in the HBV-related liver disease population. Therefore, PTX3 may be used as a simple and potentially readily available test that can accurately discriminate HCC in chronic HBV infection. Prospective studies with large patient populations of various etiologies are warranted to validate these results and to directly compare PTX3 with other noninvasive markers before establishing its diagnostic value.

Ethics approval. The study was conducted under approval of Ethics Committee of the First Affiliated Hospital of Xi'an Jiaotong University. All experiments were conducted in accordance with the Declaration of Helsinki. All the participants provided written informed consent to participate in the study.

\section{Data availability}

The data generated and analysed during this study are available from the corresponding author on reasonable request.

Received: 11 June 2020; Accepted: 6 November 2020

Published online: 20 November 2020

\section{References}

1. Bray, F. et al. Global cancer statistics 2018: GLOBOCAN estimates of incidence and mortality worldwide for 36 cancers in 185 countries. CA Cancer J. Clin. 68(6), 394-424 (2018).

2. Sangiovanni, A. et al. Increased survival of cirrhotic patients with a hepatocellular carcinoma detected during surveillance. Gastroenterology 126(4), 1005-1014 (2004).

3. Chang, M. S. \& Nguyen, M. H. Epidemiology of hepatitis B and the role of vaccination. Best Pract. Res. Clin. Gastroenterol. 31(3), 239-247 (2017).

4. Schweitzer, A., Horn, J., Mikolajczyk, R. T., Krause, G. \& Ott, J. J. Estimations of worldwide prevalence of chronic hepatitis B virus infection: a systematic review of data published between 1965 and 2013. Lancet 386(10003), 1546-1555 (2015).

5. GBD 2013 Mortality and Causes of Death Collaborators. Global, regional, and national age-sex specific all-cause and cause-specific mortality for 240 causes of death, 1990-2013: a systematic analysis for the Global Burden of Disease Study 2013. Lancet 385(9963), $117-171$ (2015).

6. El-Serag, H. B. \& Mason, A. C. Rising incidence of hepatocellular carcinoma in the United States. N. Engl. J. Med. 340(10), 745-750 (1999).

7. Zhu, Q. et al. Hepatocellular carcinoma in a large medical center of China over a 10-year period: evolving therapeutic option and improving survival. Oncotarget 6(6), 4440-4450 (2015). 
8. Forner, A., Llovet, J. M. \& Bruix, J. Hepatocellular carcinoma. Lancet 379(9822), 1245-1255 (2012).

9. Yuen, M. F. et al. Early detection of hepatocellular carcinoma increases the chance of treatment: Hong Kong experience. Hepatology 31(2), 330-335 (2000).

10. Shoreibah, M. G., Bloomer, J. R., McGuire, B. M. \& Massoud, O. I. Surveillance for hepatocellular carcinoma: evidence, guidelines and utilization. Am. J. Med. Sci. 347(5), 415-419 (2014).

11. Forner, A. \& Bruix, J. Biomarkers for early diagnosis of hepatocellular carcinoma. Lancet Oncol. 13(8), 750-751 (2012).

12. Luo, P. et al. Current status and perspective biomarkers in AFP negative HCC: towards screening for and diagnosing hepatocellular carcinoma at an earlier stage. Pathol. Oncol. Res. POR 26(2), 599-603 (2020).

13. Marrero, J. A. \& Feng, Z. Alpha-fetoprotein in early hepatocellular carcinoma. Gastroenterology 138(1), 400-401 (2010).

14. Harris, P. S. et al. Hepatocellular carcinoma surveillance: an evidence-based approach. World J. Gastroenterol. 25(13), 1550-1559 (2019).

15. Wisniewski, H. G. \& Vilcek, J. Cytokine-induced gene expression at the crossroads of innate immunity, inflammation and fertility: TSG-6 and PTX3/TSG-14. Cytokine Growth Factor Rev. 15(2-3), 129-146 (2004).

16. Bottazzi, B. et al. The long pentraxin PTX3 as a link among innate immunity, inflammation, and female fertility. J. Leukoc. Biol. 79(5), 909-912 (2006).

17. Bottazzi, B., Doni, A., Garlanda, C. \& Mantovani, A. An integrated view of humoral innate immunity: pentraxins as a paradigm. Annu. Rev. Immunol. 28, 157-183 (2010).

18. Inforzato, A. et al. PTX3 as a paradigm for the interaction of pentraxins with the complement system. Semin. Immunol. 25(1), 79-85 (2013).

19. Garlanda, C., Bottazzi, B., Bastone, A. \& Mantovani, A. Pentraxins at the crossroads between innate immunity, inflammation, matrix deposition, and female fertility. Annu. Rev. Immunol. 23, 337-366 (2005).

20. Doni, A. et al. Regulation of PTX3, a key component of humoral innate immunity in human dendritic cells: stimulation by IL-10 and inhibition by IFN-gamma. J. Leukoc. Biol. 79(4), 797-802 (2006).

21. Garlanda, C., Bottazzi, B., Magrini, E., Inforzato, A. \& Mantovani, A. PTX3, a humoral pattern recognition molecule, in innate immunity, tissue repair, and cancer. Physiol. Rev. 98(2), 623-639 (2018).

22. Doni, A. et al. The long pentraxin PTX3 as a link between innate immunity, tissue remodeling, and cancer. Frontiers Immunol. 10, 712 (2019).

23. Bonavita, E., Mantovani, A. \& Garlanda, C. PTX3 acts as an extrinsic oncosuppressor. Oncotarget 6(32), 32309-32310 (2015).

24. Jaillon, S. et al. The humoral pattern recognition molecule PTX3 is a key component of innate immunity against urinary tract infection. Immunity 40(4), 621-632 (2014).

25. Choi, B. et al. Elevated Pentraxin 3 in bone metastatic breast cancer is correlated with osteolytic function. Oncotarget 5(2), 481-492 (2014).

26. Choi, B. et al. Pentraxin-3 silencing suppresses gastric cancer-related inflammation by inhibiting chemotactic migration of macrophages. Anticancer Res. 35(5), 2663-2668 (2015).

27. Diamandis, E. P., Goodglick, L., Planque, C. \& Thornquist, M. D. Pentraxin-3 is a novel biomarker of lung carcinoma. Clin. Cancer Res. Off. J. Am. Assoc. Cancer Res. 17(8), 2395-2399 (2011).

28. Kondo, S. et al. Clinical impact of pentraxin family expression on prognosis of pancreatic carcinoma. Br. J. Cancer 109(3), 739-746 (2013).

29. Stallone, G. et al. Pentraxin 3: a novel biomarker for predicting progression from prostatic inflammation to prostate cancer. Can. Res. 74(16), 4230-4238 (2014).

30. Yoneda, M. et al. Plasma Pentraxin 3 is a novel marker for nonalcoholic steatohepatitis (NASH). BMC Gastroenterol. 8, 53 (2008).

31. Boga, S. et al. Plasma Pentraxin 3 Differentiates Nonalcoholic Steatohepatitis (NASH) from Non-NASH. Metab. Syndrome Relat. Disord. 13(9), 393-399 (2015).

32. Craig, D. G. et al. Elevated levels of the long pentraxin 3 in paracetamol-induced human acute liver injury. Eur. J. Gastroenterol. Hepatol. 25(3), 359-367 (2013).

33. Perea, L. et al. Pentraxin-3 modulates lipopolysaccharide-induced inflammatory response and attenuates liver injury. Hepatology 66(3), 953-968 (2017).

34. Gorka-Dynysiewicz, J., Pazgan-Simon, M. \& Zuwala-Jagiello, J. Pentraxin 3 detects clinically significant fibrosis in patients with chronic viral hepatitis C. Biomed. Res. Int. 2019, 2639248 (2019).

35. Carmo, R. F. et al. Genetic variation in PTX3 and plasma levels associated with hepatocellular carcinoma in patients with HCV. J. Viral Hepatitis 23(2), 116-122 (2016).

36. Song, T., Wang, C., Guo, C., Liu, Q. \& Zheng, X. Pentraxin 3 overexpression accelerated tumor metastasis and indicated poor prognosis in hepatocellular carcinoma via driving epithelial-mesenchymal transition. J. Cancer 9(15), 2650-2658 (2018).

37. European Association for the Study of the Liver. Electronic address: easloffice@easloffice.eu, \& European Association for the Study of the Liver. EASL 2017 Clinical Practice Guidelines on the management of hepatitis B virus infection. J. Hepatol. 67(2), 370-398 (2017).

38. El-Serag, H. B., Marrero, J. A., Rudolph, L. \& Reddy, K. R. Diagnosis and treatment of hepatocellular carcinoma. Gastroenterology 134(6), 1752-1763 (2008).

39. Llovet, J. M. et al. Design and endpoints of clinical trials in hepatocellular carcinoma. J. Natl Cancer Inst. 100(10), 698-711 (2008).

40. Daigo, K. et al. Pentraxins in the activation and regulation of innate immunity. Immunol. Rev. 274(1), 202-217 (2016).

41. Bottazzi, B. et al. The pentraxins PTX3 and SAP in innate immunity, regulation of inflammation and tissue remodelling. J. Hepatol. 64(6), 1416-1427 (2016).

42. Giacomini, A., Ghedini, G. C., Presta, M. \& Ronca, R. Long pentraxin 3: A novel multifaceted player in cancer. Biochimica et biophysica acta Rev. Cancer 1869(1), 53-63 (2018).

43. Bishayee, A. The role of inflammation and liver cancer. Adv. Exp. Med. Biol. 816, 401-435 (2014).

44. Lee, G. W., Lee, T. H. \& Vilcek, J. TSG-14, a tumor necrosis factor- and IL-1-inducible protein, is a novel member of the pentaxin family of acute phase proteins. J. Immunol. 150(5), 1804-1812 (1993).

45. Dinney, C. M. et al. Regulation of HBV-specific CD8(+) T cell-mediated inflammation is diversified in different clinical presentations of HBV infection. J. Microbiol. 53(10), 718-724 (2015).

46. Saxena, R., Chawla, Y. K., Verma, I. \& Kaur, J. Interleukin-1 polymorphism and expression in hepatitis B virus-mediated disease outcome in India. J. Interferon Cytokine Res. 33(2), 80-89 (2013).

47. Saxena, R., Chawla, Y. K., Verma, I. \& Kaur, J. Association of interleukin-10 with hepatitis B virus (HBV) mediated disease progression in Indian population. Indian J. Med. Res. 139(5), 737-745 (2014).

48. Xue, H. et al. Overrepresentation of IL-10-expressing B cells suppresses cytotoxic CD4+ T cell activity in HBV-induced hepatocellular carcinoma. PLoS ONE 11(5), e0154815 (2016).

49. Savchenko, A. et al. Expression of pentraxin 3 (PTX3) in human atherosclerotic lesions. J. Pathol. 215(1), 48-55 (2008).

50. Tzartzeva, K. et al. Surveillance imaging and alpha fetoprotein for early detection of hepatocellular carcinoma in patients with cirrhosis: a meta-analysis. Gastroenterology 154(6), 1706-1718.e1 (2018).

51. Chen, J., Wu, G. \& Li, Y. Evaluation of serum des-gamma-carboxy prothrombin for the diagnosis of hepatitis B virus-related hepatocellular carcinoma: a meta-analysis. Dis. Markers 2018, 8906023 (2018). 


\section{Acknowledgements}

This study was supported in part by grant from the National Natural Science Foundation of China (Grant no. 81371798). The funder plays no role in the design of study, the data collection and analysis, the preparation of the manuscript and the decision to publish.

\section{Author contributions}

Conception and design of the study: H.D., Z.L., N.L., Q.H. and Y.L. Data collection: H.D., X.F., X.W., L.Z., K.Z., X.Z., N.L. and Q.H. Statistical analysis: H.D. and N.L. Manuscript draft: H.D. and Z.L. Manuscript review and editing: H.D., X.F., X.W., L.Z., K.Z., X.Z., N.L., Q.H., Y.L. and Z.L.

\section{Funding}

National Natural Science Foundation of China (Grant No. 81371798).

\section{Competing interests}

The authors declare no competing interests.

\section{Additional information}

Supplementary information is available for this paper at https://doi.org/10.1038/s41598-020-77332-3.

Correspondence and requests for materials should be addressed to Z.L.

Reprints and permissions information is available at www.nature.com/reprints.

Publisher's note Springer Nature remains neutral with regard to jurisdictional claims in published maps and institutional affiliations.

(c) (i) Open Access This article is licensed under a Creative Commons Attribution 4.0 International License, which permits use, sharing, adaptation, distribution and reproduction in any medium or format, as long as you give appropriate credit to the original author(s) and the source, provide a link to the Creative Commons licence, and indicate if changes were made. The images or other third party material in this article are included in the article's Creative Commons licence, unless indicated otherwise in a credit line to the material. If material is not included in the article's Creative Commons licence and your intended use is not permitted by statutory regulation or exceeds the permitted use, you will need to obtain permission directly from the copyright holder. To view a copy of this licence, visit http://creativecommons.org/licenses/by/4.0/.

(C) The Author(s) 2020 